\title{
Adhesion, Permeability, and Mechanical Properties of Multilayered Blown Films Using Maleated Low-Density Polyethylene Blends as Adhesion-Promoting Layers
}

\author{
Chi-Hsien HuAnG, Jiann-Shing Wu, Chun-Chin HuAnG ${ }^{* \dagger}$ and Li-Shin LIN** \\ Department of Applied Chemistry, National Chiao Tung University, Hsinchu 300, Taiwan, R. O. C. \\ *Department of Mold and Die Engineering, National Kaohsiung University of Applied Science, \\ 415 Chien Kung Road, Kaohsiung 807, Taiwan, R. O. C. \\ ${ }^{* *}$ Chang Chun Petrochemical Co., Ltd., Miaoli 360, Taiwan, R. O. C.
}

(Received July 22, 2003; Accepted October 7, 2003)

\begin{abstract}
We have fabricated three-layer films, comprising a varying content of ethylene-vinyl alcohol copolymer (EVOH) as the internal layer and blends of low-density polyethylene (LDPE) and low-density ethylene grafted with maleic anhydride (LDPE- $g$-MAH) as the external layers, by a coextrusion blown-film process. We chose to use blends to promote the adhesion between EVOH and LDPE and to reduce the number of layers in the coextrusion system. The peel strength increased sharply when the amount of LDPE-g-MAH was greater than $12.5 \mathrm{wt} \%$ and we associate it with the promotion of adhesion between layers brought about by specific interactions between EVOH and LDPE- $g$-MAH. FT-IR spectroscopic analysis showed an increase in the intensity of the absorbance of the ester band upon increasing the content of LDPE- $g$-MAH, which indicates that a chemical reaction occurs that promotes adhesion at the interface. The tensile strength did not change significantly with increasing LDPE- $g$-MAH content, which had a small effect on elongation and modulus in both the machine direction (MD) and transverse direction (TD). Tear strength decreased continuously with increasing LDPE- $g$-MAH content, in both the MD and TD, as a result of the greater ease of crack propagation in the EVOH layer. The oxygen permeability of the three-layer films remained almost constant upon varying the amount of LDPE- $g$ MAH. These three-layer films all followed the theoretical prediction made by the inverse additivity rule. The water vapor permeabilities of the three-layer films, however, were affected by the degree of hydrogen bonding, which was analyzed by FT-IR spectroscopy. The effects of this hydrogen bonding resulted in a discrepancy between the experimental findings and the theoretical predictions, especially when the EVOH content was increased.

KEY WORDS Multilayered Film / Coextrusion / EVOH / Blending / Hydrogen Bonding / Barrier Properties /
\end{abstract}

Coextrusion is a process in which two or more polymers are extruded simultaneously and joined together to form a single structure having different properties in each layer and to achieve a broad range of properties that are not available in any of the individual materials alone. In recent years, the packaging and container industries have paid increasing attention to the development of new or improved products formed by coextrusion, such as multilayer sheets, multilayer films, and multilayer containers. ${ }^{1-3}$ The number of layers comprising these materials depends on the required end-use properties and the availability of polymer combinations suitable for specific applications.

Recently, it has become common ${ }^{4,5}$ in food packaging technology to coextrude multilayer films consisting of distinct layers that are barriers for oxygen and moisture. Polyethylene is an excellent moisture barrier for packaging, and its low cost, strength and ease of processing make it suitable for many applications. Its inability, however, to act as a barrier for oxy- gen, aromatics, and oils limits its potential applications. On the other hand, ethylene-vinyl alcohol copolymer $(\mathrm{EVOH})$ possesses excellent barrier properties to oxygen, aromatics, and oils. ${ }^{6-9}$ Unfortunately, EVOH is highly sensitive to moisture, which alters its ability to acts as an oxygen barrier. ${ }^{10,11}$ Therefore, using coextrusion to combine polyethylene and EVOH in a multilayer structure is very attractive for many demanding packaging applications, such as for food, drugs, and cosmetics. Typical commercial multilayer barrier films for food packaging contain EVOH as an oxygen barrier layer and polyethylene resins as the moisture barrier layer. This film possesses a multilayer structure in which outer PE layers protect an inner EVOH layer from continuous exposure to moisture. Because of the chemical dissimilarities between $\mathrm{PE}$ and $\mathrm{EVOH}$, however, an extrudable adhesive polymer must be incorporated into the film as a tie layer that promotes adhesion. Graft copolymers are widely recognized as novel potential adhesive polymers for imparting improved

${ }^{\dagger}$ To whom all correspondence should be addressed (Tel: +886-7-3814526 ext2700, Fax: +886-7-3835015, E-mail: chhuang.ac87g@nctu.edu.tw). 
adhesion. These copolymers are synthesized mainly by modifying polyolefin resins through the addition of functionality. This process is achieved by adding acid or anhydride units to polyolefins through grafting or by direct synthesis of copolymers. Tanaka et al. ${ }^{12}$ have successfully developed a new generation of tie layer adhesives, by combining graft and polymer blending, that maintain high adhesive strengths after thermoforming and orientation. Botros studied three-layer films, tie/EVOH/tie, using a coextrusion cast-film process ${ }^{13}$ and found that the tie layers bind to EVOH through covalent and hydrogen bonding. The failure mechanisms of the three-layer films were of a mixed cohesive/adhesive type. Kim et al. ${ }^{14}$ have investigated the mechanical and transport properties of various combinations of two-layer films, including LDPE/tie, Nylon 6/tie and LDPE/Nylon 6. The tensile strength and modulus of a coextruded film follows the additivity rule and its permeability follows the inverse additivity rule. ${ }^{15}$ Kamykowski studied the adhesive properties of fivelayer coextruded cast films ${ }^{16}$ and found that the adhesion properties generally improved upon increasing the overall film thickness or the relative amount of the adhesive (maleic anhydride-grafted polypropylene). The molecular weight of the grafted resin had a small effect on adhesion. Homopolymer diluents outperform random copolymer diluents in their adhesion properties.

Having additional tie layers in a coextruded film makes the fabrication process more complex and expensive, because of the need in the coextrusion system for a specially designed die and additional extruders for the adhesive polymers. An alternative approach has been reported that overcomes this disadvantage by replacing the five-layer film coextrusion system with a three-layer film comprising EVOH as the central layer and LLDPE/LLDPE- $g$-MAH blends as the external layers. ${ }^{17,18}$

In this paper, we report a method to eliminate the need for tie layers by using blends of low-density polyethylene (LDPE) and linear low-density polyethylene grafted with maleic anhydride (LDPE-g-MAH) that promote adhesion between LDPE and EVOH in coextruded three-layer blown films. We investigated the mechanical properties of the films, including their peel strengths, tensile properties, and tear strengths, and compared their oxygen and water vapor permeability to theoretical predictions. ${ }^{19}$ These blown films could be a viable option for reducing the number of film layers in coextrusion processing.

\section{EXPERIMENTAL}

\section{Materials}

Commercial-grade, low-density polyethylene [LDPE, 6030F, M. I. $\left(\mathrm{g} / 10 \mathrm{~min}, 190^{\circ} \mathrm{C}, 2.16 \mathrm{~kg}\right)=$ 0.27 , density $\left.=0.922 \mathrm{~g} \mathrm{~cm}^{-3}\right]$ was supplied in pellet form by Formosa Plastic Corp. (Taiwan). The ethylene-vinyl alcohol copolymer [EVOH, F101A, ethylene content $(\mathrm{mol} \%)=32$, M. I. $\left(\mathrm{g} / 10 \mathrm{~min}, 190^{\circ} \mathrm{C}\right.$, $2.16 \mathrm{~kg})=1.6$, density $\left.=1.19 \mathrm{~g} \mathrm{~cm}^{-3}\right]$ was provided in pellet form by Kuraray Co. (Japan). The adhesive, Modic-AP L502, was obtained in pellet form from Mitsubishi Chemical Corp. (Japan). It is a low-density polyethylene-grafted maleic anhydride [LDPE-g$\mathrm{MAH}, \mathrm{M}$. I. $\left(\mathrm{g} / 10 \mathrm{~min}, 190^{\circ} \mathrm{C}, 2.16 \mathrm{~kg}\right)=1.0$, density $=$ $\left.0.93 \mathrm{~g} \mathrm{~cm}^{-3}\right]$.

\section{Preparing Blends}

LDPE- $g$-MAH was dried in a vacuum oven for $24 \mathrm{~h}$ period at $50{ }^{\circ} \mathrm{C}$ before blending. Blends of LDPE with different amounts of LDPE- $g$-MAH $(0,5,7.5,10$, $12.5,15,20$, and $25 \mathrm{wt} \%$ ) were prepared in pellet form by melt mixing in a twin-screw extruder operating at $210^{\circ} \mathrm{C}$ and $40 \mathrm{rpm}$.

\section{Preparing Multilayer Films}

Prior to processing, EVOH was dried in a vacuum oven for $24 \mathrm{~h}$ at $60{ }^{\circ} \mathrm{C}$. The extruded blends (the external layers) were coextruded with EVOH (the internal layer) through a three-layer coextrusion blown-film die (inner diameter $=97.6 \mathrm{~mm}$; gap thickness $=1.2 \mathrm{~mm}$ ) at $230^{\circ} \mathrm{C}$. A $42-\mathrm{mm}$-diameter extruder $(\mathrm{L} / \mathrm{D}=28)$ was used for the extruded blends and a 25 -mm-diameter extruder $(\mathrm{L} / \mathrm{D}=28)$ was used for $\mathrm{EVOH}$. All extruders were attached with gear pumps to control the thickness of individual component layers precisely. The ratios of take-off and blow-up were 3.5 and 2.5, respectively. Three-layer films were fabricated with $\mathrm{EVOH}$ as the internal layer (overall $16.6 \mathrm{wt} \%$ ) and blends of LDPE and LDPE- $g$-MAH $(0,5,7.5,10,12.5,15,20$, or $25 \mathrm{wt} \%$ of LDPE) as the external layers (overall $83.4 \mathrm{wt} \%$ ). The thickness of all the three-layer films produced, irrespective of the relative amount of LDPE- $g$-MAH, was $150 \mu \mathrm{m}$ (blend, $130 \mu \mathrm{m}$; EVOH, $20 \mu \mathrm{m}$ ). We also prepared three-layer films having overall EVOH content of $7.9,13,17.1,21.2$ or $24.4 \mathrm{wt} \%$ (internal layer) and a blend of LDPE with $15 \mathrm{wt} \%$ of LDPE- $g$-MAH (external layers). Table I shows the thicknesses of these three-layer films with respect to the EVOH content. To allow theoretical predictions to be made, we also prepared monolayer film samples of LDPE, EVOH, and 
LDPE- $g$-MAH under the same processing conditions.

\section{Preparing Specimens for Measuring Peel Strengths}

Film samples were cut into strips $(25 \mathrm{~mm} \times 300 \mathrm{~mm})$ and delamination was initiated at one end by soaking the tips of each strip in formic acid ( $\mathrm{HCOOH})$ for $c a$. $4 \mathrm{~h}$. The corner of a strip was pulled repeatedly with tweezers until we observed a slight indication of delamination. The delamination was then propagated throughout the width of the strip. The peel strengths of the three-layer films were measured at $23^{\circ} \mathrm{C}$ by $\mathrm{T}$ type using a tensile tester with a crosshead speed of $25.4 \mathrm{~cm} \mathrm{~min}^{-1}$, following the procedure described in ASTM D1876. At least ten samples were tested to obtain average values.

\section{Analysis by FT-IR Spectroscopy}

The interactions at the interface between the layers of the blend and EVOH were analyzed by Fourier transform infrared (FT-IR) spectroscopy as a function of the content of LDPE- $g$-MAH and EVOH using a Bio-Rad FTS-165. At least 32 scans at a resolution of $4 \mathrm{~cm}^{-1}$ were signal-averaged. The hydrogen bonding was also analyzed by FT-IR spectroscopy.

\section{Measuring Tensile Properties}

The tensile properties of the three-layer films, including tensile strength, tensile modulus, and ultimate elongation, were measured by a tensile tester at $23{ }^{\circ} \mathrm{C}$ and a relative humidity of $50 \%$ in both the machine direc-

Table I. Thickness of individual layers of three-layer films with various $\mathrm{EVOH}$ contents

\begin{tabular}{ccc}
\hline Sample No. & $\begin{array}{c}\text { Composition }(w t \%) \\
\text { Blend } / \text { EVOH }\end{array}$ & $\begin{array}{c}\text { Layer Thickness }(\mu \mathrm{m}) \\
\text { Blend/EVOH }\end{array}$ \\
\hline 1 & $92.1 / 7.9$ & $136 / 9$ \\
2 & $87 / 13$ & $130 / 15$ \\
3 & $82.9 / 17.1$ & $125 / 20$ \\
4 & $78.8 / 21.2$ & $120 / 25$ \\
5 & $75.6 / 24.4$ & $116 / 29$ \\
\hline
\end{tabular}

${ }^{\mathrm{a}}$ Blend $=$ LDPE $(85 \mathrm{wt} \%) /$ LDPE- $g$-MAH $(15 \mathrm{wt} \%)$. tion (MD) and the transverse direction (TD), following the procedure described in ASTM D882. At least ten samples were tested to obtain average values.

\section{Measuring Tear Strength}

The tear strengths of the three-layer films were measured by a tensile tester at $23{ }^{\circ} \mathrm{C}$ and a relative humidity of $50 \%$ in both the machine direction (MD) and the transverse direction (TD), following the procedure described in ASTM D1938. At least ten samples were tested to obtain average values.

\section{Measuring Oxygen Permeability}

The oxygen permeability properties of the threelayer films were measured using a Lyssy L-1005000 Gas Permeability Tester ${ }^{20}$ following the ASTM D1434. The oxygen permeability of the films was measured at $23{ }^{\circ} \mathrm{C}$ and a relative humidity of $0 \%$. The temperature was controlled by a water bath.

\section{Measuring Water Vapor Permeability}

Water vapor permeability properties of the threelayer films were measured using Lyssy L-80-5000 Water Vapor Permeability Tester, ${ }^{21}$ following the ASTM E96. The water vapor permeability of the films was measured at $38^{\circ} \mathrm{C}$ and a relative humidity of $90 \%$. The temperature was controlled by a water bath.

\section{RESULTS AND DISCUSSION}

Table II lists the tensile, tear and barrier properties of individual component materials. It is seen that the LDPE-g-MAH exhibit almost the same values in all mechanical and barrier properties as LDPE. The EVOH has much better tensile strength, modulus, tear strength, and oxygen barrier property than both LDPE and LDPE-g-MAHH. On the other hand, LDPE and LDPE- $g$-MAH exhibit higher tensile elongation and water vapor barrier property. We assume that these properties of the blends are not affected significantly

Table II. Properties of films for individual component materials

\begin{tabular}{cccc}
\hline \multirow{2}{*}{ Property } & \multicolumn{3}{c}{ Material } \\
\cline { 2 - 4 } & $\begin{array}{c}\text { LDPE } \\
(130 \mu \mathrm{m})\end{array}$ & $\begin{array}{c}\text { LDPE- } g \text {-MAH } \\
(130 \mu \mathrm{m})\end{array}$ & $\begin{array}{c}\mathrm{EVOH} \\
(20 \mu \mathrm{m})\end{array}$ \\
\hline Tensile Strength MD/TD (MPa) & $24.2 / 21.4$ & $23.6 / 20.6$ & $63 / 45$ \\
Tensile Elongation MD/TD (\%) & $670 / 720$ & $690 / 750$ & $280 / 210$ \\
Tensile Modulus MD/TD (MPa) & $205 / 220$ & $220 / 235$ & $1590 / 1610$ \\
Tear Strength MD/TD (N) & $9.9 / 12$ & $10.3 / 12.4$ & $<0.1 /<0.1$ \\
$\begin{array}{c}\text { Oxygen Permeability }{ }^{\mathrm{a}} \\
\left(\mathrm{mL}-\mathrm{mm} / \mathrm{m}^{2}-24 \text { h-atm) } \times 10^{2}\right.\end{array}$ & 1300 & 1250 & 11 \\
Water Vapor Permeability & & & \\
$\left(\mathrm{g}-\mathrm{mm} / \mathrm{m}^{2}-24 \mathrm{~h}\right) \times 10^{2}$ & 9 & 8.7 & 700 \\
\hline${ }^{\mathrm{a}} 23^{\circ} \mathrm{C}, 50 \%$ relative humidity. ${ }^{\mathrm{b}} 38^{\circ} \mathrm{C}, 90 \%$ relative humidity.
\end{tabular}




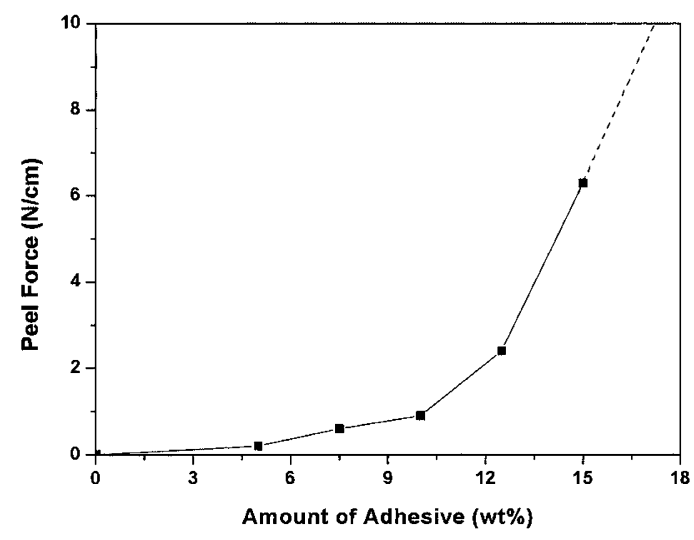

Figure 1. Peel strength between LDPE/LDPE- $g$-MAH blend and $\mathrm{EVOH}$ of three-layer films as a function of LDPE- $g$-MAH content.

by the relative amount of LDPE-g-MAH in LDPE due to the same level of properties as shown in Table II.

\section{Peel Properties}

Figure 1 shows the peel strength as a function of the relative amount of LDPE- $g$-MAH. The peel strength increased slightly upon increasing the amount of LDPE$g$-MAH and then increased sharply when the amount of LDPE- $g$-MAH was greater than $12.5 \mathrm{wt} \%$. When the LDPE- $g$-MAH content was above $15 \mathrm{wt} \%$, we observed in the three-layer films a concomitant elongation and break in the blend layer of the film in addition to peeling at the interface between layers of EVOH and blend. As mentioned above, this observation indicates that good adhesion exists between the blend and $\mathrm{EVOH}$ layers and, as a result, it changes the failure mechanism from adhesive to cohesive.

The interactions at the interface between the blend and EVOH layers were analyzed by FT-IR spectroscopy as shown in Figure 2. Table III lists the absorbances of the ester bands near $1710 \mathrm{~cm}^{-1}$ and the hydroxyl bands near $3350 \mathrm{~cm}^{-1}$. We observe a continuous increase in the intensity of the ester absorbance and a decrease in that of the hydroxyl absorbance upon increasing the amount of LDPE-g-MAH. The increase in the ester band absorbance is attributed to covalent bonding between the carbonyl groups in LDPE- $g$-MAH and the hydroxyl groups in EVOH. These stronger interactions lead to an increase in the adhesion between the blend and EVOH layers, which is a property supported by the increasing peel strengths shown in Figure 1.

\section{Tensile Properties}

Figure 3 shows the tensile properties in both the machine direction (MD) and the transverse direction (TD) as a function of the amount of LDPE- $g$-MAH ( 0 to $25 \mathrm{wt} \%$ ). In Figure 3 (a), we see that the tensile strength

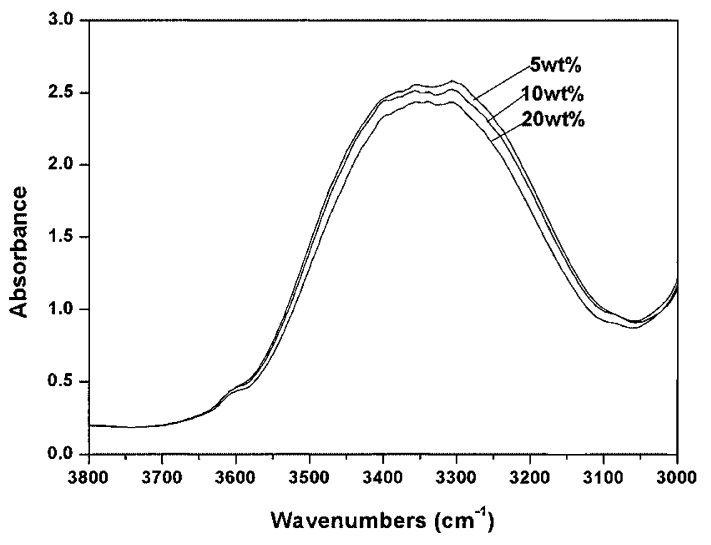

(a)

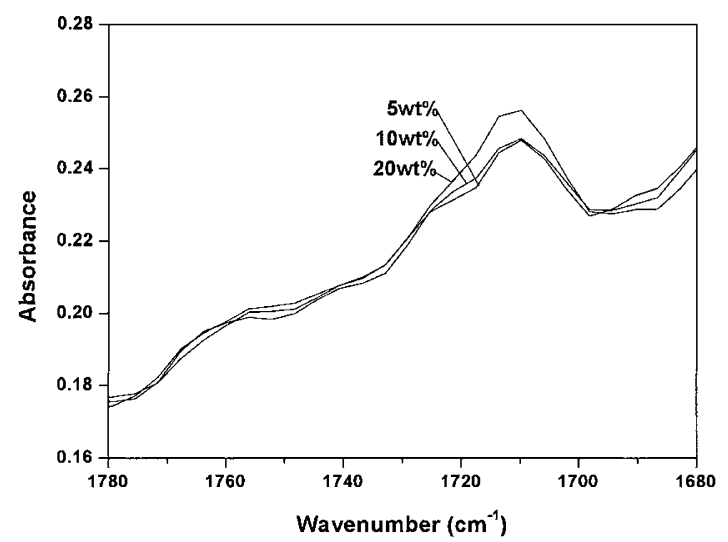

(b)

Figure 2. IR absorption spectra of three-layer films at the (a) hydroxyl band; (b) ester band with various LDPE- $g$-MAH contents.

Table III. IR absorbance of hydroxyl and ester bands of three-layer films with different LDPE-g-MAH contents

\begin{tabular}{ccc}
\hline $\begin{array}{c}\text { LDPE- } g \text {-MAH content } \\
(\mathrm{wt} \%)\end{array}$ & $\begin{array}{c}\text { Hydroxyl Band } \\
3350 \mathrm{~cm}^{-1}\end{array}$ & $\begin{array}{c}\text { Ester Band } \\
1710 \mathrm{~cm}^{-1}\end{array}$ \\
\hline 0 & 623.9 & 0.461 \\
5 & 610.2 & 0.547 \\
7.5 & 600.7 & 0.583 \\
10 & 592.8 & 0.616 \\
12.5 & 587 & 0.671 \\
15 & 575.7 & 0.714 \\
20 & 560.1 & 0.789 \\
\hline
\end{tabular}

does not change significantly in either the MD or the TD upon changing the relative amount of LDPE- $g$ MAH. The tensile strengths of three-layer films, however, are all lower than those of the films of the individual materials (see table II). These results are much different from those reported for three-layer films of LDPE/tie/PS ${ }^{15}$ and two-layer films of LDPE/tie, Nylon 6/tie and LDPE/Nylon $6 .{ }^{14}$ In these previous studies, the tensile strengths of the two- or three-layer films were found to follow the additivity rule. ${ }^{15}$ Figure $3(\mathrm{~b})$ shows that the elongation at break increases slightly as the relative amount of LDPE- $g$-MAH increases. It is believed that high-elongation materials, such as LDPE 


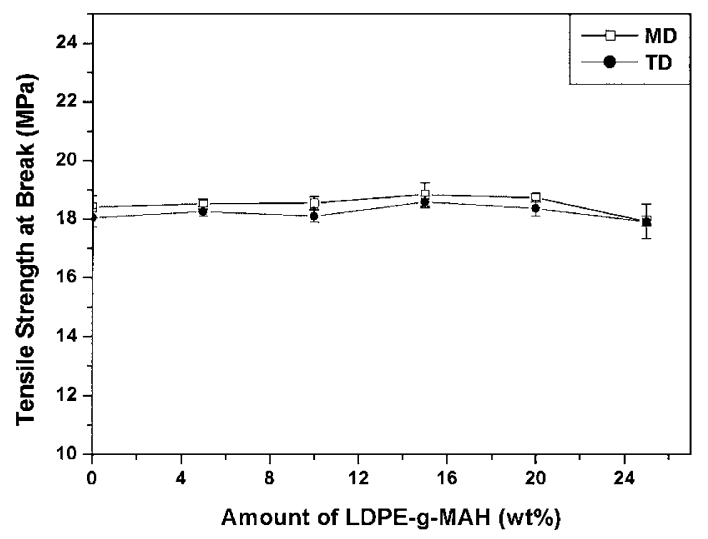

(a)

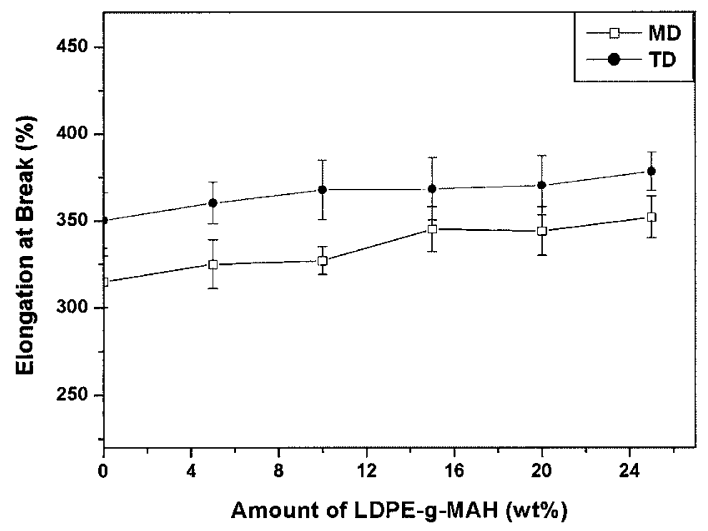

(b)

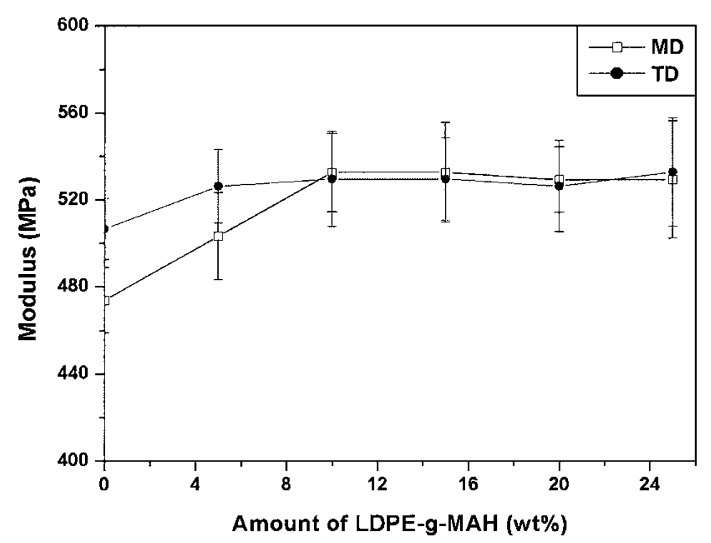

(c)

Figure 3. Tensile Properties of three-layer films as a function of LDPE-g-MAH content for both MD and TD: (a) strength at break; (b) elongation at break; (c) modulus.

or LDPE-g-MAH, as shown in Table II, block transverse crack propagation in the EVOH layer, which causes the elongation to be about twice that of the separate single EVOH film in both the MD and TD. ${ }^{15}$ Figure $3(\mathrm{c})$ shows that the tensile modulus increases initially_ — up to $10 \mathrm{wt} \%$ for the MD and $5 \mathrm{wt} \%$ for the TD — with increasing the relative amount of LDPE- $g$ $\mathrm{MAH}$, and then it stays constant This property is due to the much higher tensile modulus of EVOH, as shown in Table III, which leads to improved tensile moduli for the three-layer films as a result of strong adhesion be-

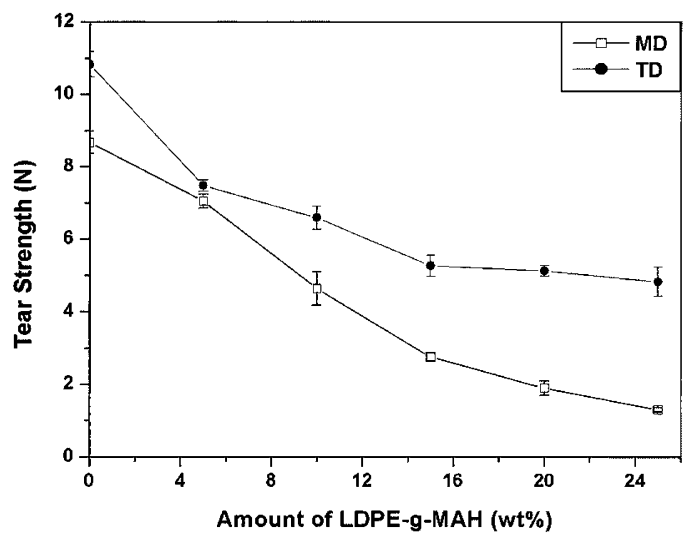

Figure 4. Tear strength of three-layer films as a function LDPE- $g$-MAH content for both MD and TD.

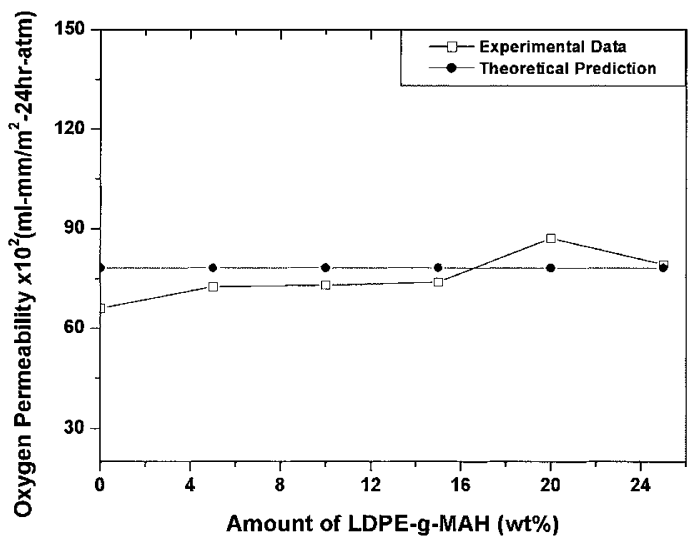

Figure 5. Oxygen permeabilities of three-layer films as a function of LDPE- $g$-MAH content.

tween LDPE and EVOH.

\section{Tear Properties}

Figure 4 presents the tear strengths of the three-layer films investigated in both the MD and TD. A continuous decrease in tear strength is observed upon increasing the relative amount of LDPE- $g$-MAH. This property is due to the poor tear resistance of $\mathrm{EVOH}$ (see Table II) and the increase in adhesion, i.e., the increase of the degree of chemical bonding. The crack propagation in EVOH continues relatively easily and this failure mechanism transfers to the blend layers because of the chemical bonding in the three-layer film structures. Thus, the better the adhesion, the easier it becomes for crack growth to occur through the entire three-layer film, which results in lower tear strengths.

\section{Oxygen Permeability}

Figure 5 presents the oxygen permeabilities of the three-layer films as a function of the relative amount of LDPE- $g$-MAH, and shows that it is almost constant. For the sake of comparison, this figure also shows the theoretical predictions of oxygen permeabilities using 


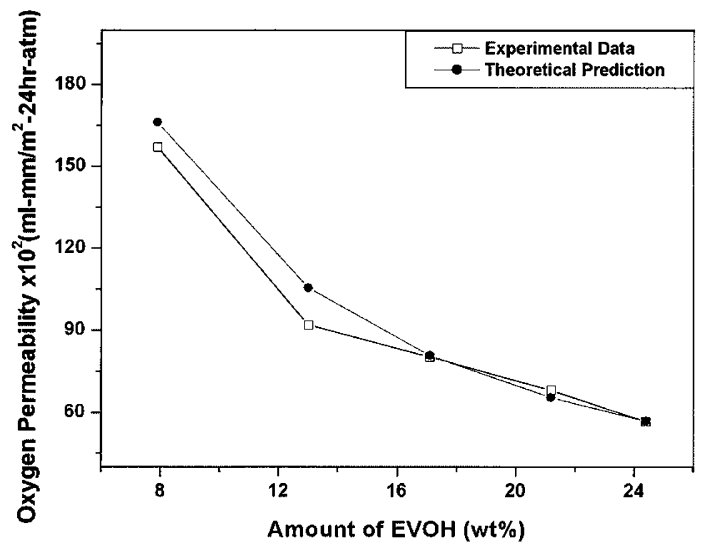

Figure 6. Oxygen permeability of three-layer films as a function of EVOH content.

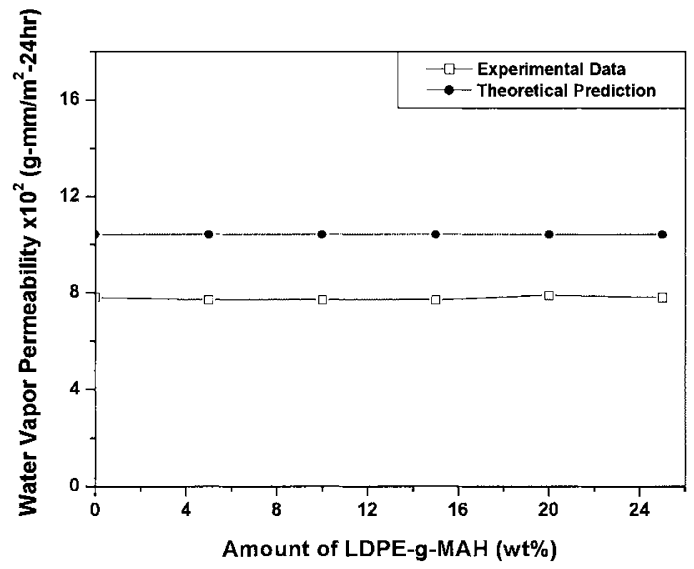

Figure 7. Water vapor permeability of three-layer films as a function of LDPE- $g$-MAH content.

the inverse additivity rule. ${ }^{19}$

$$
\frac{1}{P_{\text {three-layer }}}=\frac{\varphi_{\text {blend }}}{P_{\text {blend }}}+\frac{\varphi_{\text {EVOH }}}{P_{E V O H}}
$$

where $\varphi$ is the volume fraction and $P$ is the oxygen permeability. Table II lists the oxygen permeabilities of the individual component materials. A good agreement exists between the experimental and theoretical data. Figure 6 displays the experimental and theoretical oxygen permeabilities of the three-layer films as a function of EVOH content. As expected, the permeability decreases with increasing the relative amount of EVOH, and it agrees reasonably well with the theoretical prediction.

\section{Water Vapor Permeability}

Figures 7 and 8 present the water vapor permeabilities of the three-layer films as a function of the relative amounts of LDPE-g-MAH and EVOH, respectively. For the sake of comparison, the two figures also show the theoretical predictions made by eq 1 and data of individual component materials (see Table II). In Figure 7, we see that the water vapor permeability of the three-layer films is almost constant with respect

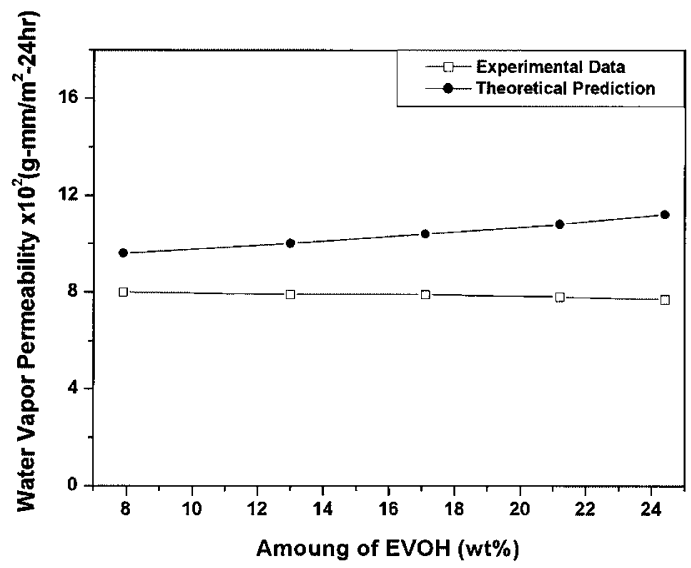

Figure 8. Water vapor permeability of three-layer films as a function of EVOH content.

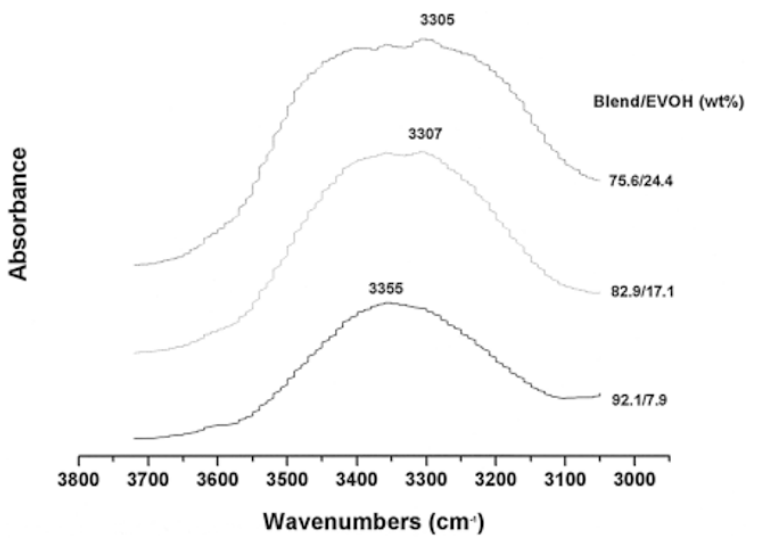

Figure 9. IR absorption spectra of three-layer films at the hydroxyl band with various $\mathrm{EVOH}$ contents.

to the LDPE- $g$-MAH content, but a significant discrepancy exists between the experimental data and the theoretical prediction. Figure 8 shows that upon increasing the EVOH content the water vapor permeability of the three-layer films decreases slightly and the discrepancy between the experimental data and theoretical prediction becomes larger. From these observations, we conclude that a constant relative amount of EVOH leads to an almost constant discrepancy between the experimental and theoretical data, but that increasing levels of EVOH result in larger discrepancies. This feature may be due to the increased amount of intramolecular hydrogen bonding in the EVOH layer, which allows barely any water vapor to pass through the three-layer films. Figure 9 shows the IR absorption spectra, viewed in the region of the hydroxyl band, of the three-layer films as a function of EVOH content. With increasing EVOH content, the absorption peaks of the hydroxyl bands near $3350 \mathrm{~cm}^{-1}$ shift towards lower wavenumbers as a result of the increasing degree of intramolecular hydrogen bonding. ${ }^{22,23}$ The magnitude of the band shift is proportional to the strength of hydrogen bonding. The increased strength of hydrogen bonding makes 
it more difficult for water vapor to pass through the films, and results in a larger discrepancy observed between the experimental data and the theoretical prediction.

\section{CONCLUSIONS}

We have fabricated three-layer films, consisting of EVOH as the internal layer and blends of low-density polyethylene and low-density ethylene grafted with maleic anhydride (LDPE- $g$-MAH) as external layers, by a coextruded blown-film process. By investigating the adhesion, permeability and mechanical properties, we make the following conclusions: (1) The adhesion of three-layer films, consisting of incompatible layers, can be improved by using blends of maleated lowdensity polyethylene and low-density polyethylene as a result of hydrogen bond formation, which can be analyzed by FT-IR spectroscopy. (2) The tensile strength does not change with increasing LDPE- $g$-MAH content, but it results in a slight increase in elongation of both the MD and TD. The modulus is only slightly increased at low levels of the LDPE-g-MAH content, and is almost constant in the MD and TD when the amount of LDPE- $g$-MAH is greater than 10 and $5 \mathrm{wt} \%$, respectively. (3) The tear strengths in both the MD and TD decrease significantly with increasing LDPE- $g$-MAH content as a result of the greater ease of crack propagation in $\mathrm{EVOH}$ that is transferred to the entire three-layer film by the improved adhesion. (4) The oxygen permeabilities of the three-layer films follow the inverse additivity rule upon varying the EVOH and LDPE- $g$ MAH contents. The water vapor permeabilities of the three-layer films, however, did not follow this rule because increasing degrees of hydrogen bonding result in reduced permeability.

\section{REFERENCES}

1. D. K. Lee, U. S. Patent 6544661 (Apr. 8, 2003).

2. T. J. Kraft, E. P. Socci, and M. K. Akkapeddi, U. S. Patent 200 3045640 (Mar. 6, 2003).

3. H. Siewert and M. Thielen, Kunst. Plast. Euro., 88, 48 (1998).

4. F. Hensen, Plastics Extrusion Technology, Hanser, New York N.Y., 1997.

5. A. P. Sullivan, E. Baer, A. Hiltner, G. J. Castle, C. E. Bibbons, and I. D. Sand, U. S. Patent 2002150704 (Oct. 17, 2002).

6. R. H. Foster, Packaging, 32, 70 (1987).

7. T. Iwanami and Y. Hirai, Tappi J., 66, 1404 (1983).

8. J. M. Lagaron, A. K. Powell, and G. Bonner, Polym. Test., 20, 569 (2001).

9. H. U. Beckmann and Ch. Herschbach, Kunst. Plast. Euro., 86, 5 (1996).

10. Z. Zhang, I. J. Britt, and M. A. Tung, J. Appl. Polym. Sci., 82, 1866 (2001).

11. R. Gopalakrishnan, J. M. Schultz, and R. Gohil, J. Appl. Polym. Sci., 56, 1749 (1995).

12. H. Tanaka, H. Shigemoto, and H. Kawachi, J. Plast. Film Sheet., 12, 279 (1996).

13. M. G. Botros, J. Plast. Film Sheet., 12, 195 (1996).

14. Y. J. Kim, C. D. Han, B. K. Song, and E. Kouassi, J. Appl. Polym. Sci., 29, 2359 (1984).

15. W. J. Schrenk and T. Alfrey, Polym. Eng. Sci., 9, 393 (1969).

16. G. W. Kamykowski, J. Plast. Film Sheet., 16, 237 (2000).

17. S. S. Valdes, F. O. Villarreal, M. L. Quintanilla, I. Y. Flores, and L. F. Ramos de Valle, Polym. Eng. Sci., 38, 127 (1998).

18. J. V. Olmos, S. S. Valdes, and I. G. Yánez Flores, Polym. Eng. Sci., 39, 1597 (1999).

19. J. B. Faisant, A. Aït-Kadi, M. Bousmina, and L. Deschênes, Polymer, 39, 533 (1998).

20. Automatic Manometric Gas Permeability Tester Operator Manual, Model L100-5000, Lyssy AG (2001).

21. Automatic Manometric Water Vapor Permeability Tester Operator Manual, Model L800-5000, Lyssy AG (2001).

22. L. Dai and L. Ying, Macromol. Mater. Eng., 287, 509 (2002).

23. J. C. Speakman, "The Hydrogen Bond and Other Intermolecular Forces", The Chemical Society, London, 1975. 